\title{
Anisotropic Diffusion in Galactic Cosmic Ray transport using PICARD
}

\author{
R. Kissmann ${ }^{a}$, O. Reimer ${ }^{\star a}$, F. Niederwanger ${ }^{a}$, and A. W. Strong ${ }^{b}$ \\ ${ }^{a}$ Institut für Astro- und Teilchenphysik, Leopold-Franzens-Universität Innsbruck, Austria \\ ${ }^{b}$ Max-Planck-Institut für extraterrestrische Physik \\ E-mail: ralf.kissmann@uibk.ac.at, olaf.reimereuibk.ac.at, \\ Felix.Niederwanger@uibk.ac.at, aws@mpe.mpg.de
}

\begin{abstract}
The Picard code for the numerical solution of the cosmic ray propagation problem is optimised to incorporate the complexity of the 3D structure of our Galaxy down to decaparsec scales. Picard was used to investigate the impact of anisotropic cosmic-ray diffusion on the Galactic cosmic-ray flux distribution. We discuss different magnetic-field models for our Galaxy in the context of a cosmic-ray source distribution aligned with the Galactic spiral arms. With the resulting sets of transport parameters, that can reproduce cosmic-ray observations at Earth for the different magnetic field models, we find restrictions for the spatial diffusion tensor. We show that related variations in the diffusive coupling to the Galactic halo can lead to changes in the cosmic-ray gradients. The most severe changes are found in the Galactic centre where a modification in the magnetic-field topology can change the cosmic-ray proton flux by more than a factor of two.
\end{abstract}

35th International Cosmic Ray Conference - ICRC2017

10-20 July, 2017

Bexco, Busan, Korea

\footnotetext{
* Speaker.
} 


\section{Introduction}

The discovery of cosmic rays dates back more than 100 years, with current research being principally devided into three major fields: heliospheric, Galactic, and extragalactic cosmic rays. Of these only heliospheric cosmic rays offer the possibility to obtain simultaneous observations at different locations within the heliosphere, leading to tight constraints for the related propagation models. In the case of Galactic cosmic rays, direct observations are only possible at a single point in space and also in time. Some information about the spatial distribution of cosmic rays in the Galaxy, can only be inferred indirectly from observation of photons generated by interactions of cosmic rays with the Galactic environment, i.e. synchrotron and gamma-ray emission (see $[24 ; 1]$ ). This is part of the reason why numerical modelling of cosmic-ray transport is more advanced for the heliosphere than for Galactic cosmic rays.

For Galactic cosmic-ray transport modelling the transition from simplified axisymmetric models to those that allow a fully three-dimensional variation of all transport parameters has just begun recently (see $[12 ; 15 ; 22 ; 31 ; 21]$ ). This reflects the important impact of the three-dimensional structure of our Galaxy, becoming relevant because of the interaction between the cosmic rays and the interstellar medium. Here, the most obvious is the source distribution of the cosmic-rays (see, e.g. $[12 ; 8 ; 22 ; 31])$ and the distribution of interstellar matter relevant for energy losses, spallation processes and different gamma-ray production channels (see [28; 1]).

One aspect that is still often neglected in models of Galactic cosmic ray transport is the influence of the magnetic field. While it is certainly acknowledged in the production of synchrotron emission [24] and corresponding energy losses it is also the magnetic field that is essential in producing the effect of diffusive transport. Basic theory states that diffusion should be anisotropic with regard to the direction of the magnetic field $[19 ; 26]$. For the heliosphere it was found that parallel and perpendicular diffusion indeed need to vary independently to allow a reproduction of observations - especially at different heliographic latitudes [25]. For Galactic cosmic rays spatial diffusion has largely been assumed to be isotropic and position independent [28]. The few notable exceptions discussed the impact of spatially variable diffusion $[13 ; 17]$ and also of spatially anisotropic diffusion [12]. The latter study found a strong impact of anisotropic diffusion on the spatial variation of the Galactic cosmic-ray flux. Here, we will also investigate the case of anisotropic diffusion, but with a focus on different structures for the Galactic magnetic field.

\section{Model Description}

In our numerical modelling efforts, we solve the Galactic cosmic-ray transport equation

$$
\begin{aligned}
\frac{\partial j_{i}}{\partial t}= & q(\vec{r}, p)+\nabla \cdot\left(\mathscr{D} \nabla j_{i}-\vec{u} j_{i}\right)+\frac{\partial}{\partial p} p^{2} D_{p p} \frac{\partial}{\partial p} \frac{1}{p^{2}} j_{i} \\
& -\frac{\partial}{\partial p}\left\{\dot{p} j_{i}-\frac{p}{3}(\nabla \cdot \vec{u}) j_{i}\right\}-\frac{1}{\tau_{f}} j_{i}-\frac{1}{\tau_{r}} j_{i}
\end{aligned}
$$

for each cosmic-ray species $i$ using the propagation code PICARD [20]. Here, $j_{i}$ is the cosmic-ray flux for species $i, q$ is the source distribution, $\mathscr{D}$ is the energy-dependent tensor of spatial diffusion, $\vec{u}$ is the advection velocity, $D_{p p}$ is the momentum diffusion coefficient, $\dot{p}$ represents all energy losses, and $\tau_{f}$ and $\tau_{r}$ are the timescales for fragmentation and radioactive decay, respectively. 
To model the cosmic-ray sources we use a distribution function peaking at the presumed position of the Galactic spiral arms within a model of a four-arm galaxy taken from [27] (for details on our implementation see [21]). Here, we start from a set a propagation parameters that was adapted to reproduce cosmic-ray observations at Earth for spatially constant, isotropic diffusion in the context of such a spiral-arm source distribution model. The diffusion tensor in that model is:

$$
\mathscr{D}=D_{0} \hat{1} \quad \text { with } \quad D_{0}=\tilde{D}_{0} \beta\left(\frac{\rho}{\rho_{0}}\right)^{\delta}
$$

with $\hat{1}$ the unit matrix, $\tilde{D}_{0}=6.5 \cdot 10^{24} \mathrm{~m}^{2} \mathrm{~s}^{-1}, \delta=0.33$ and the reference rigidity $\rho_{0}=4 \mathrm{GV}$. In this study we use $\vec{u}=0$. All other relevant parameters can be found in [21]. In all models discussed here the transport equation was solved for all nuclei up to Silicon, with a focus on low mass nuclei up to Nitrogen. The solution was computed on a Cartesian mesh with extent $x, y=-20 \ldots 20 \mathrm{kpc}$ and $z=-4 \ldots 4 \mathrm{kpc}$ using 257 grid points in $x$ and $y$ and 65 in $z$. We consider energies from 0.01 up to $10^{6} \mathrm{GeV}$, where we cover the range with 127 logarithmically distributed grid points.

\subsection{The Diffusion Tensor}

In the following we focus on the impact of a non-isotropic spatial diffusion. In that case we assume the diffusion tensor to have the shape:

$$
\mathscr{D}=\left(\begin{array}{ccc}
D_{\|} & 0 & 0 \\
0 & D_{\perp} & 0 \\
0 & 0 & D_{\perp}
\end{array}\right)
$$

in the local coordinate system of the magnetic field, with the field pointing in the $x$-direction. A magnetic field pointing in the arbitrary direction $\vec{n}$ then implies a general diffusion tensor: with components

$$
\begin{array}{ll}
D_{x x}=D_{\|} \cos ^{2} \theta \sin ^{2} \phi+D_{\perp}\left(\cos ^{2} \phi+\sin ^{2} \theta \sin ^{2} \phi\right) & \\
D_{y y}=D_{\|} \cos ^{2} \theta \cos ^{2} \phi+D_{\perp}\left(\sin ^{2} \phi+\sin ^{2} \theta \cos ^{2} \phi\right) & \\
D_{z z}=D_{\|} \sin ^{2} \theta+D_{\perp} \cos ^{2} \theta & D_{x y}=\left(D_{\perp}-D_{\|}\right) \cos ^{2} \theta \sin \phi \cos \phi \\
D_{x z}=\left(D_{\perp}-D_{\|}\right) \sin \theta \cos \theta \sin \phi & D_{y z}=\left(D_{\perp}-D_{\|}\right) \sin \theta \cos \theta \cos \phi .
\end{array}
$$

where $\theta$ is the angle between $\vec{B}$ and the Cartesian $z$-direction and $\phi$ is the angle between the projection of $-\vec{B}$ on the $x y$-plane and the Cartesian $x$-direction. In the following, we assume two different models for the magnetic field. The first model uses a magnetic field with $B_{z}=0$, and correspondingly $\theta=90^{\circ}$, with field lines along spiral arms with a pitch-angle $\alpha=14.17^{\circ}$. The second one uses a physically motivated magnetic field structure also featuring a magnetic field component in the vertical direction.

Observations revealed that several external Galaxies show a prominent X-shape for the magnetic field perpendicular to the Galactic plane [18]. These are thought to be related to the galactic wind outflows. [14] introduced a range of analytical magnetic field models that have the potential to reproduce the corresponding observations in the radio regime. For this the authors combined a spiral-arm magnetic-field model in the Galactic plane with an X-shape structure in the vertical direction. Here, we use their model Dd presumably being the most realistic of the given models. For an illustration of the field structure within the numerical domain used in this study see Fig. 1. 


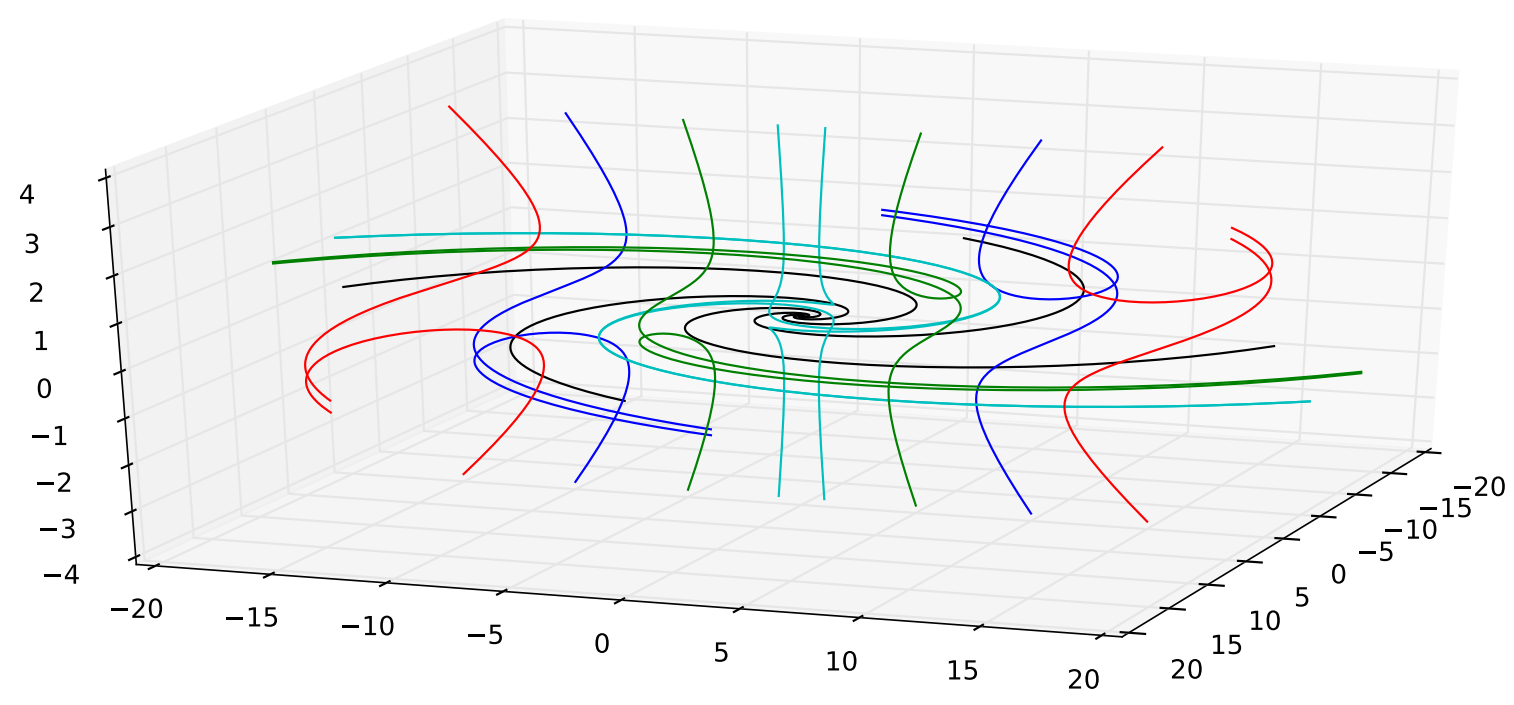

Figure 1: Illustration of the Galactic magnetic field model taken from [14] and described in Sec. 2.1. Field lines in the Galactic plane are shown in black. Additionally, magnetic field lines connecting to the upper or lower boundary of the numerical domain are shown. These are conneted to the boundary of the domain at $1 \mathrm{kpc}$ (cyan), $5 \mathrm{kpc}$ (green), $10 \mathrm{kpc}$ (blue), and $15 \mathrm{kpc}$ (red). The vertical dimension is stretched by a factor of 2 to aid visibility.

\section{Results}

In all our models with anisotropic diffusion we use a ratio of parallel to perpendicular diffusion of 10. For spiral arm magnetic field models, we distinguish between a low-diffusion model with $D_{\|}=D_{0}$ and $D_{\perp}=D_{0} / 10$ and a high-diffusion model with $D_{\|}=10 D_{0}$ and $D_{\perp}=D_{0}$. Compared to a model with isotropic diffusion, the low diffusion model shows more pronounced spiral arms due to the decreased diffusion perpendicular to the spiral arms, i.e. gradients in the direction perpendicular to the spiral arms have become distinctively steeper. This is the same behaviour as was also observed and extensively investigated by [12]. In contrast to those authors we also took re-acceleration, i.e. momentum diffusion, into account in order to find a good correspondence to the relevant cosmic-ray observations. In our case the decrease in perpendicular diffusion leads to significant changes to the proton spectrum as compared to the one with isotropic diffusion (see Fig. 2 on the left). Corresponding secondary to primary ratios are also found to be in disagreement with the data for the low diffusion model. Thus, by simply reducing the perpendicular diffusion the simulation results do not agree with observations anymore.

Results computed with the high-diffusion mode are in agreement with the data at a similar level as the isotropic model, for which the propagation parameters were initially adapted. This is to be expected because spatial diffusion perpendicular the spiral arms is the same as for the isotropic diffusion model. Along the spiral arms the gradients in the source distribution are rather small. Thus, an increased diffusion along this direction only leads to small changes in the resulting cosmic-ray flux. This also shows that this is the only model with a simple spiral-arm magnetic field geometry that is compatible with the data. In such a magnetic-field geometry anisotropic diffusion does not lead to significant changes in the cosmic-ray flux anywhere in the Galaxy. 

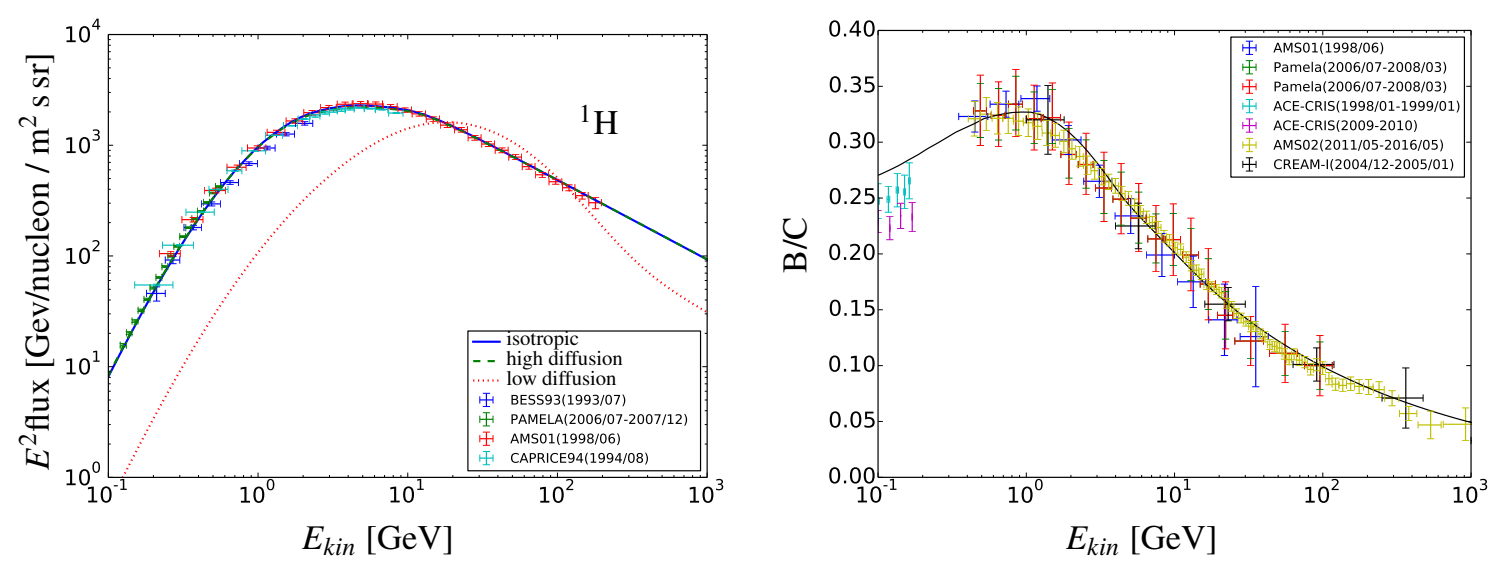

Figure 2: Predicted cosmic-ray flux at the nominal position of Earth. On the left the total proton flux (using a modulation potential of $450 \mathrm{MV}$ ) is shown for a model with a spiral-arm magnetic field. On the right the $\mathrm{B} / \mathrm{C}$ ratio is shown for our model with an $\mathrm{X}$-shape magnetic field, where we used a modulation potential of 350 MV. Proton data are taken from [7] (AMS-01), [30] (BESS), [9] (CAPRICE), and [2] (Pamela). B/C data are taken from [11] and [23] (ACE), [4] (AMS-1), [5] (AMS-2), [6] (CREAM), and [3] (Pamela).

This is different, when considering a magnetic-field model with $B_{z} \neq 0$. For the field Dd from [14] (see Sec. 2.1) it is possible to find agreement with the data for rather different propagation parameters than in the isotropic diffusion case as shown on the right in Fig. 2. In producing these results for B/C, the following propagation parameters were adapted: $\tilde{D}_{0}=2.1 \cdot 10^{24} \mathrm{~m}^{2} \mathrm{~s}^{-2}$ and $v_{A}=3 \cdot 10^{4} \mathrm{~m} \mathrm{~s}^{-1}$, where $D_{\|}=10 D_{0}$ and $D_{\perp}=D_{0}$ in this case.

This magnetic-field model has a distinct impact on the spatial distribution of the cosmic rays, illustrated by the distribution of ${ }^{12} \mathrm{C}$ for an energy of $\sim 1 \tilde{\mathrm{GeV}}$ in Fig. 3. In this case the structure of the spiral arms becomes more pronounced, also leading to larger gradients in secondary-to-primary ratios as discussed in [21]. In the direction vertical to the Galactic plane the $\mathrm{X}$-shape magnetic field obviously introduces additional structure to the cosmic-ray distribution. Especially in the Galactic center the cosmic-ray flux is considerably reduced, up to a factor of 2.5 for secondaries, as compared to models with isotropic diffusion. This is caused by the structure of the magnetic field, that bends away from the Galactic center. The effect is particularly strong here, since the sources in the current model are restricted to the spiral arms and sources in the Galactic center, e.g. related to a Galactic bar, are not taken into account.

\section{Discussion}

We investigated the impact of anisotropic diffusion on the transport of Galactic cosmic rays, where diffusion was higher along the local magnetic-field direction. In our model the ratio of parallel to perpendicular diffusion was set to 10 without any energy dependence. With this we modelled transport for a galaxy with a spiral shaped magnetic field structure without a vertical component of the magnetic field and for a galaxy with a spiral-shape magnetic field model together with an X-shape halo magnetic field.

In the former model we found that the only setup compatible with observational data is an increased diffusion along the magnetic field, when a cosmic-ray source distribution peaking at the 

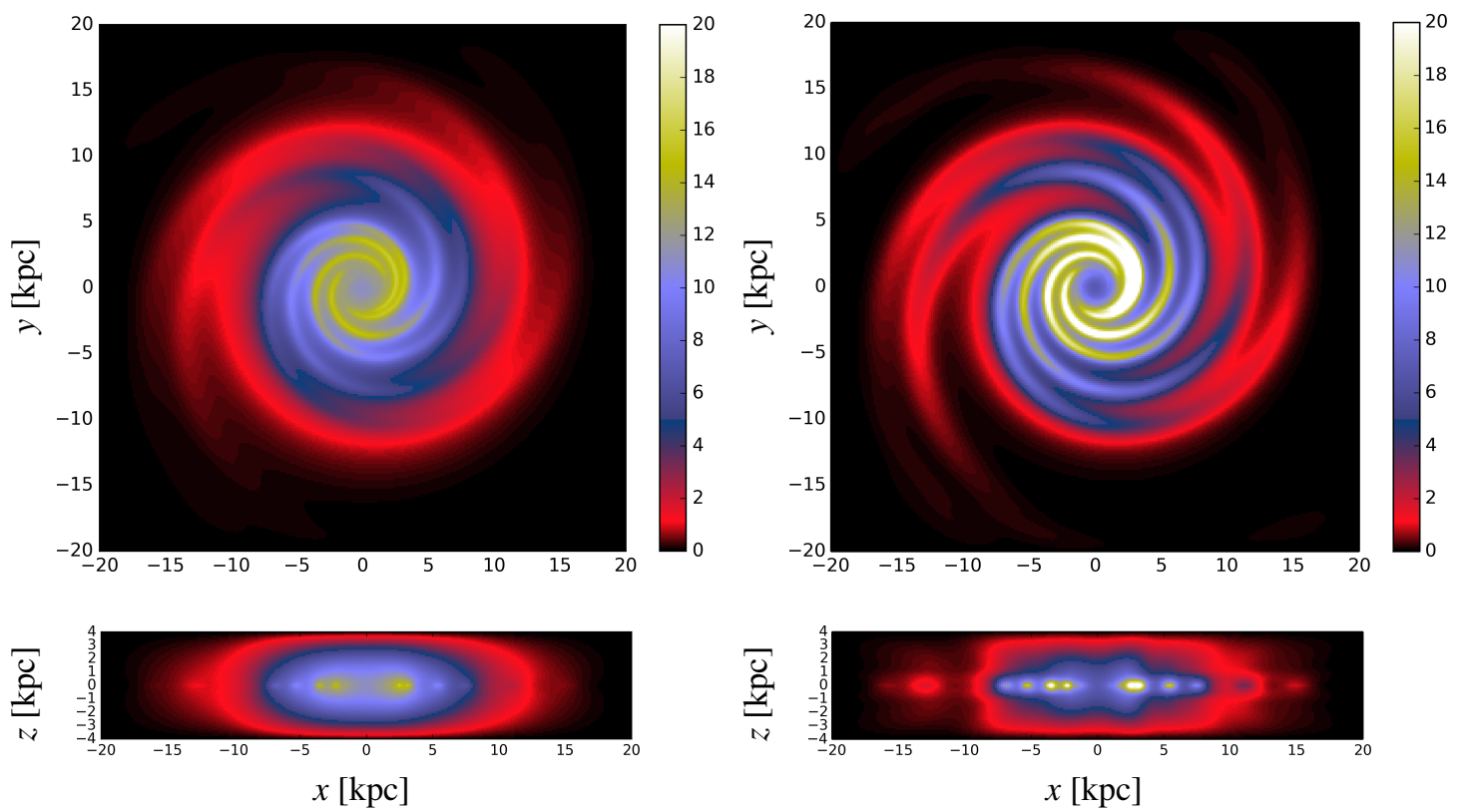

Figure 3: Propagation results for the $\sim 1 \mathrm{GeV}$ per nucleon ${ }^{12} \mathrm{C}$ flux in the Galactic plane (top) and the $x-z$ plane (bottom). On the left results are shown for isotropic diffusion and on the right for anisotropic diffusion along an X-shape magnetic-field model.

Galactic spiral arms is assumed. This, however, produces only negligible changes to the cosmicray flux throughout the model galaxy. The impact of anisotropic diffusion, however, would become more prominent, when using individual localised sources instead of a smooth source distribution. This is a task that can be tackled with the PICARD code in the future, because of its capability to solve the transport equation in a high-resolution 3D setup.

In contrast, the $\mathrm{X}$-shape model shows distinct changes in the resulting cosmic-ray flux. Reproducing the cosmic-ray flux observed at Earth requires a corresponding change in the propagation parameters, where $D_{\perp}<D_{0}^{\text {iso }}<D_{\|}$, with $D_{0}^{\text {iso }}$ the strength of diffusion in the isotropic model, because the diffusion in the vertical direction, that critically determines the cosmic-ray flux near Earth, is determined by parallel and perpendicular diffusion in this model. This situation changes near the Galactic center, where field lines are nearly vertical. This leads to a lower flux in the Galactic center, underlining the special role of the Galactic-center transport also discussed in modelling efforts of the Galactic-center gamma-ray excess (see. e.g. [10; 16]. The increased diffusion vertical to the Galactic plane also might help to explain the origin of the Fermi bubbles, large-scale structures of increased gamma-ray flux above and below the Galactic enter [29]. In conclusion, a re-evaluation of this excess in the context of these anisotropic diffusion models becomes necessary.

\section{References}

[1] M. Ackermann, et al. Fermi-LAT Observations of the Diffuse $\gamma$-Ray Emission: Implications for Cosmic Rays and the Interstellar Medium. ApJ 750, 3.

[2] O. Adriani, et al. Measurement of the Isotopic Composition of Hydrogen and Helium Nuclei in Cosmic Rays with the PAMELA Experiment. ApJ 770, 2. 1304.5420. 
[3] O. Adriani, et al. Measurement of Boron and Carbon Fluxes in Cosmic Rays with the PAMELA Experiment. ApJ 791, 93. 1407.1657.

[4] M. Aguilar, et al. Relative Composition and Energy Spectra of Light Nuclei in Cosmic Rays: Results from AMS-01. ApJ 724, (2010) 329.

[5] M. Aguilar, et al. Precision Measurement of the Proton Flux in Primary Cosmic Rays from Rigidity 1 GV to 1.8 TV with the Alpha Magnetic Spectrometer on the International Space Station. Physical Review Letters 114 (17), 171103.

[6] H. S. Ahn, et al. Measurements of cosmic-ray secondary nuclei at high energies with the first flight of the CREAM balloon-borne experiment. Astroparticle Physics 30, (2008) 133. 0808.1718.

[7] J. Alcaraz, et al. Cosmic protons. Physics Letters B 490, (2000) 27.

[8] D. Benyamin, et al. Recovering the Observed B/C Ratio in a Dynamic Spiral-armed Cosmic Ray Model. ApJ 782, 34. 1308.1727.

[9] M. Boezio, et al. The Cosmic-Ray Proton and Helium Spectra between 0.4 and 200 GV. ApJ 518, (1999) 457.

[10] E. Carlson, T. Linden, and S. Profumo. Improved cosmic-ray injection models and the Galactic Center gamma-ray excess. Phys. Rev. D 94 (6), 063504. 1603.06584.

[11] G. A. de Nolfo, et al. Observations of the $\mathrm{Li}, \mathrm{Be}$, and B isotopes and constraints on cosmic-ray propagation. Advances in Space Research 38, (2006) 1558. arXiv: astro-ph/ 0611301.

[12] F. Effenberger, et al. Anisotropic diffusion of Galactic cosmic ray protons and their steadystate azimuthal distribution. A\&A 547, A120. 1210.1423.

[13] C. Evoli, et al. Common Solution to the Cosmic Ray Anisotropy and Gradient Problems. Physical Review Letters 108 (21), 211102. 1203.0570.

[14] K. Ferrière and P. Terral. Analytical models of X-shape magnetic fields in galactic halos. A\&A 561, A100. 1312.1974.

[15] D. Gaggero, et al. Three-Dimensional Model of Cosmic-Ray Lepton Propagation Reproduces Data from the Alpha Magnetic Spectrometer on the International Space Station. Physical Review Letters 111 (2), 021102. 1304.6718.

[16] D. Gaggero, et al. Towards a realistic astrophysical interpretation of the gamma-ray Galactic center excess. J. Cosmology Astropart. Phys. 12, 056. 1507.06129.

[17] D. Gaggero, et al. Diffuse cosmic rays shining in the Galactic center: A novel interpretation of H.E.S.S. and Fermi-LAT gamma-ray data. ArXiv e-prints 1702.01124.

[18] V. Heesen, et al. Cosmic rays and the magnetic field in the nearby starburst galaxy NGC 253. II. The magnetic field structure. A\&A 506, (2009) 1123. 0801.3542. 
[19] J. R. Jokipii. Cosmic-Ray Propagation. I. Charged Particles in a Random Magnetic Field. ApJ 146, (1966) 480.

[20] R. Kissmann. PICARD: A novel code for the Galactic Cosmic Ray propagation problem. Astroparticle Physics 55, (2014) 37. 1401.4035.

[21] R. Kissmann, et al. Propagation in 3D spiral-arm cosmic-ray source distribution models and secondary particle production using PICARD. Astroparticle Physics 70, (2015) 39. 1504.08249 .

[22] A. Kopp, et al. A stochastic approach to Galactic proton propagation: Influence of the spiral arm structure. New A 30, (2014) 32.

[23] K. A. Lave, et al. Galactic Cosmic-Ray Energy Spectra and Composition during the 20092010 Solar Minimum Period. ApJ 770, 117.

[24] E. Orlando and A. Strong. Galactic synchrotron emission with cosmic ray propagation models. MNRAS 436, (2013) 2127. 1309.2947.

[25] M. Potgieter. Solar Modulation of Cosmic Rays. Living Reviews in Solar Physics 10, (2013) 3. 1306.4421 .

[26] R. Schlickeiser. Cosmic-ray transport and acceleration. I - Derivation of the kinetic equation and application to cosmic rays in static cold media. II - Cosmic rays in moving cold media with application to diffusive shock wave acceleration. ApJ 336, (1989) 243.

[27] T. Y. Steiman-Cameron, M. Wolfire, and D. Hollenbach. COBE and the Galactic Interstellar Medium: Geometry of the Spiral Arms from FIR Cooling Lines. ApJ 722, (2010) 1460.

[28] A. W. Strong, I. V. Moskalenko, and V. S. Ptuskin. Cosmic-Ray Propagation and Interactions in the Galaxy. Annual Review of Nuclear and Particle Science 57, (2007) 285. arXiv:astro-ph/0701517.

[29] M. Su, T. R. Slatyer, and D. P. Finkbeiner. Giant Gamma-ray Bubbles from Fermi-LAT: Active Galactic Nucleus Activity or Bipolar Galactic Wind? ApJ 724, (2010) 1044. 1005.5480.

[30] J. Z. Wang, et al. Measurement of Cosmic-Ray Hydrogen and Helium and Their Isotopic Composition with the BESS Experiment. ApJ 564, (2002) 244.

[31] M. Werner, et al. Spiral arms as cosmic ray source distributions. Astroparticle Physics 64, (2015) 18. 1410.5266. 